PROCEEDINGS OF THE

AMERICAN MATHEMATICAL SOCIETY

Volume 140, Number 3, March 2012, Pages 779-793

S 0002-9939(2011)11150-X

Article electronically published on July 14, 2011

\title{
THE $K$-THEORETIC FARRELL-JONES CONJECTURE FOR CAT(0)-GROUPS
}

\author{
CHRISTIAN WEGNER
}

(Communicated by Alexander N. Dranishnikov)

\begin{abstract}
We prove the $K$-theoretic Farrell-Jones conjecture with (twisted) coefficients for $\mathrm{CAT}(0)$-groups.
\end{abstract}

\section{INTRODUCTION}

The $K$-theoretic Farrell-Jones conjecture with coefficients for a group $G$ says that the $K$-theoretic assembly map

$$
H_{m}^{G}\left(E_{\mathcal{V} C y c} G ; \mathbf{K}_{\mathcal{A}}\right) \rightarrow H_{m}^{G}\left(\mathrm{pt} ; \mathbf{K}_{\mathcal{A}}\right) \cong K_{m}\left(\int_{G} \mathcal{A}\right)
$$

is an isomorphism for all $m \in \mathbb{Z}$ and every additive category $\mathcal{A}$ with a strict right $G$-action. Here $E_{\mathcal{V} \mathcal{C}_{y c}} G$ denotes the classifying space of the group $G$ with respect to the family of virtually cyclic subgroups. Any additive category $\mathcal{A}$ with a right $G$ action induces a covariant functor $\mathbf{K}_{\mathcal{A}}$ from the orbit category of $G$ to the category of spectra with (strict) maps of spectra as morphisms (see [BR07, Definition 3.1]). We denote the associated $G$-homology theory by $H_{*}^{G}\left(-; \mathbf{K}_{\mathcal{A}}\right)$ (see DL98, sections 4 and 7]). The assembly map is the map induced by the projection $E_{\mathcal{V C} y c} G \rightarrow \mathrm{pt}$ onto the space consisting of one point.

The $K$ - and $L$-theoretic Farrell-Jones conjectures play an important role in the classification and geometry of manifolds. Moreover, they imply a variety of wellknown conjectures, e.g. the Bass, Borel, Kaplansky and Novikov conjectures. For more information on the Farrell-Jones conjectures we refer to the survey article LR05.

In this paper we prove the $K$-theoretic Farrell-Jones conjecture with coefficients for $\mathrm{CAT}(0)$-groups. By a CAT(0)-group we mean a group which admits a cocompact proper action by isometries on a finite-dimensional CAT(0)-space. The proof is based on methods from BLR08, BL09 and BL10. In BLR08 Bartels, Lück and Reich show the bijectivity of the $K$-theoretic assembly map for hyperbolic groups. In [BL09] and [BL10] Bartels and Lück investigate the $K$-theoretic assembly map for CAT(0)-groups and prove bijectivity in degree $m \leq 0$ and surjectivity in degree $m=1$.

Received by the editors December 15, 2010.

2000 Mathematics Subject Classification. Primary 19D10; Secondary 19A31, 19B28, 20F67.

Key words and phrases. Farrell-Jones conjecture, algebraic $K$-theory of group rings, CAT(0)groups.

The work on this paper was supported by the SFB 878 - Groups, Geometry \& Actions.

(C)2011 American Mathematical Society 
The general strategy to prove the $K$-theoretic Farrell-Jones conjecture is to study the obstruction category $\mathcal{O}^{G}\left(E_{\mathcal{F}} G, \mathrm{pt} ; \mathcal{A}\right)$ whose $K$-theory gives the homotopy fiber of the $K$-theoretic assembly map. Then a transfer map has to be constructed which allows us to replace the one-point space by a suitable metric space which gives room for certain constructions. This metric space has to be carefully chosen since we need contracting properties afterwards. In the case of hyperbolic groups this space is a compactification of the Rips complex of the group $G$. In the case of CAT $(0)$-groups we use large closed balls in the associated CAT(0)-space. Finally, contractible maps on the metric spaces are used to gain control and to prove the vanishing of the $K$-theory groups of the obstruction category.

The main difficulty in enlarging the result of Bartels and Lück comes from the fact that the closed balls in the CAT(0)-space are no $G$-spaces. They only admit a homotopy $G$-action. This is sufficient to define the transfer map for $K_{1}$ since this map only requires homotopy chain actions. But for higher $K$-theory we have to take account of higher homotopies. A useful tool to tackle this problem is strong homotopy actions which we introduce in section 2 They describe in a simple way a homotopy action together with all higher homotopies. We use them in section 3 where we define the notion of strong transfer reducibility for groups. This definition specifies the requirements that we have on the metric space which replaces the onepoint space. We show that hyperbolic groups and CAT(0)-groups are strongly transfer reducible over the family of virtually cyclic subgroups (see Example 3.2 and Theorem 3.4).

The following sections are dedicated to the proof of the $K$-theoretic Farrell-Jones conjecture with coefficients for groups which are strongly transfer reducible. More precisely, we prove

Theorem 1.1. Let $G$ be a group which is strongly transfer reducible over a family $\mathcal{F}$ of subgroups of $G$. Let $\mathcal{A}$ be an additive $G$-category, i.e. an additive category with a strict right $G$-action by functors of additive categories. Then the $K$-theoretic assembly map

$$
H_{m}^{G}\left(E_{\mathcal{F}} G ; \mathbf{K}_{\mathcal{A}}\right) \rightarrow H_{m}^{G}\left(\mathrm{pt} ; \mathbf{K}_{\mathcal{A}}\right) \cong K_{m}\left(\int_{G} \mathcal{A}\right)
$$

is an isomorphism for all $m \in \mathbb{Z}$.

In section 4 we give a short review of controlled algebra which is a crucial tool in the proof. In particular, we define the obstruction category. An outline of the proof of Theorem 1.1 is given in section 5] The last two sections deal with the transfer map and finish the proof of Theorem 1.1 .

Following the proof of BL10, Lemma 2.3] we see that Theorem 1.1 and Theorem 3.4 imply

Corollary 1.2. Let $G_{1}, G_{2}$ be groups which satisfy the K-theoretic Farrell-Jones conjecture with coefficients. Then the groups $G_{1} \times G_{2}$ and $G_{1} * G_{2}$ satisfy the $K$-theoretic Farrell-Jones conjecture with coefficients, too.

\section{Strong homotopy ACtions}

Let $G$ be a $\mathrm{CAT}(0)$-group, i.e. a group which admits a cocompact proper action by isometries on a finite-dimensional CAT(0)-space $Y$. We would like to replace the CAT(0)-space $Y$ by a compact space, namely a large ball in $Y$. The price we 
have to pay for this replacement is that we only retain a $G$-action on the ball up to homotopy. To control these homotopies we introduce the notion of a strong homotopy action.

Definition 2.1. A strong homotopy action of a group $G$ on a topological space $X$ is a continuous map

$$
\Psi: \coprod_{j=0}^{\infty}\left((G \times[0,1])^{j} \times G \times X\right) \rightarrow X
$$

with the following properties:

(1) $\Psi\left(\ldots, g_{l}, 0, g_{l-1}, \ldots\right)=\Psi\left(\ldots, g_{l}, \Psi\left(g_{l-1}, \ldots\right)\right)$,

(2) $\Psi\left(\ldots, g_{l}, 1, g_{l-1}, \ldots\right)=\Psi\left(\ldots, g_{l} \cdot g_{l-1}, \ldots\right)$,

(3) $\Psi\left(e, t_{j}, g_{j-1}, \ldots\right)=\Psi\left(g_{j-1}, \ldots\right)$,

(4) $\Psi\left(\ldots, t_{l}, e, t_{l-1}, \ldots\right)=\Psi\left(\ldots, t_{l} \cdot t_{l-1}, \ldots\right)$,

(5) $\Psi\left(\ldots, t_{1}, e, x\right)=\Psi(\ldots, x)$,

(6) $\Psi(e, x)=x$.

A strong homotopy action restricts to a homotopy $S$-action in the sense of BL09, Definition 1.4] by setting $\phi_{g}(x):=\Psi(g, x)$ and $H_{g, h}(x, t):=\Psi(g, t, h, x)$. But the strong homotopy action also perceives the higher homotopies. The simple description of a strong homotopy action is very useful for our purpose.

Remark 2.2. It is not true in general that a topological space $X$ which is homotopy equivalent to a $G$-space admits a strong homotopy action by conjugation with the homotopy equivalence.

Strong homotopy actions appear in the following situation: Let $Y$ be a $G$-space and let $H: Y \times[0,1] \rightarrow Y$ be a deformation retraction onto a subspace $X \subseteq Y$ (i.e. $H_{0}(Y)=X,\left.H_{0}\right|_{X}=\operatorname{id}_{X}$ and $H_{1}=\operatorname{id}_{Y}$ ) such that $H_{t} \circ H_{t^{\prime}}=H_{t \cdot t^{\prime}}$ for all $t, t^{\prime} \in[0,1]$. For example, we can consider the $\mathrm{CAT}(0)$-space together with a deformation retraction on a ball by projecting along geodesics. (We will make this more precise in the proof of Theorem 3.4.) In this situation we define

$$
\Omega: \coprod_{j=0}^{\infty}\left((G \times[0,1])^{j} \times G \times X\right) \rightarrow Y
$$

inductively by $\Omega\left(g_{0}, x\right):=g_{0} \cdot x$ and $\Omega\left(g_{j}, t_{j}, g_{j-1}, \ldots\right):=g_{j} \cdot H_{t_{j}}\left(\Omega\left(g_{j-1}, \ldots\right)\right)$ for $j \geq 1$. Then $\Psi:=H_{0} \circ \Omega$ is a strong homotopy action.

On the other hand, a strong homotopy action $\Psi$ induces a subspace $M$ of the space of continuous mappings $\coprod_{j=0}^{\infty}(G \times[0,1])^{j} \times G \rightarrow X$. It is defined by

$$
M:=\left\{\Psi\left(?, t_{\alpha}, g_{\alpha-1}, \ldots, g_{0}, x\right) \mid \alpha \in \mathbb{N}_{0}, t_{i} \in[0,1], g_{i} \in G, x \in X\right\}
$$

and has a $G$-action given by $c_{g}(f):=f(?, 1, g)$. Moreover, we obtain a deformation retraction $H: M \times[0,1] \rightarrow M, f \mapsto f(?, t, e)$ onto the subspace $H_{0}(M)=\{\Psi(?, x) \mid$ $x \in X\} \cong X$ which satisfies $H_{t} \circ H_{t^{\prime}}=H_{t \cdot t^{\prime}}$ for all $t, t^{\prime} \in[0,1]$.

If we start with a strong homotopy action and construct the associated deformation retraction, then the strong homotopy action associated to this deformation retraction coincides with the original strong homotopy action. In general, the other composition of the two constructions is not the identity. Nevertheless, both 
constructions are inverse to each other if the interior $\stackrel{\circ}{X}^{\circ}$ of $X=H_{0}(Y)$ satisfies $G \cdot \stackrel{X}{X}=Y$. This condition is satisfied in the case of our CAT(0)-group as long as the ball, on which we project, is large enough. Anyhow, we will not make use of this fact.

In analogy to BL09, Definition 1.4 and Definition 3.4] we make the following definition.

Definition 2.3. Let $\Psi$ be a strong homotopy $G$-action on a metric space $\left(X, d_{X}\right)$. Let $S \subseteq G$ be a finite symmetric subset which contains the trivial element $e \in G$. Let $k \in \mathbb{N}$ be a natural number.

(1) For $g \in G$ we define $F_{g}(\Psi, S, k) \subset \operatorname{map}(X, X)$ by

$F_{g}(\Psi, S, k):=\left\{\Psi\left(g_{k}, t_{k}, \ldots, g_{0}, ?\right): X \rightarrow X \mid g_{i} \in S, t_{i} \in[0,1], g_{k} \cdot \ldots \cdot g_{0}=g\right\}$.

(2) For $(g, x) \in G \times X$ we define $S_{\Psi, S, k}^{1}(g, x) \subset G \times X$ as the subset consisting of all $(h, y) \in G \times X$ with the following property: There are $a, b \in S$, $f \in F_{a}(\Psi, S, k)$ and $\tilde{f} \in F_{b}(\Psi, S, k)$ such that $f(x)=\tilde{f}(y)$ and $h=g a^{-1} b$. For $n \in \mathbb{N} \geq 2$ we set

$$
S_{\Psi, S, k}^{n}(g, x):=\left\{S_{\Psi, S, k}^{1}(h, y) \mid(h, y) \in S_{\Psi, S, k}^{n-1}(g, x)\right\} .
$$

(3) For $\Lambda \in \mathbb{R}^{>0}$ we define the quasi-metric $d_{\Psi, S, k, \Lambda}$ on $G \times X$ as the largest quasi-metric on $G \times X$ satisfying

- $d_{\Psi, S, k, \Lambda}((g, x),(g, y)) \leq \Lambda \cdot d_{X}(x, y)$ for all $g \in G, x, y \in X$ and

- $d_{\Psi, S, k, \Lambda}((g, x),(h, y)) \leq 1$ for all $(h, y) \in S_{\Psi, S, k}^{1}(g, x)$.

We remind the reader that the difference between a metric and a quasi-metric is that in the later case the distance $\infty$ is allowed. Notice that the quasi-metric $d_{\Psi, S, k, \Lambda}$ is $G$-invariant with respect to the $G$-action $g(h, x):=(g h, x)$ on $G \times X$. See BL09, Definition 3.4] for a construction of the quasi-metric $d_{\Psi, S, k, \Lambda}$. The following lemma is taken from BL09, Lemma 3.5].

Lemma 2.4. (1) The subset $S$ generates $G$ if and only if $d_{\Psi, S, k, \Lambda}$ is a metric.

(2) Let $(g, x),(h, y) \in G \times X$ and $n \in \mathbb{N}$. Then $(h, y) \in S_{\Psi, S, k}^{n}(g, x)$ if and only if $d_{\Psi, S, k, \Lambda}((g, x),(h, y)) \leq n$ for all $\Lambda>0$.

(3) The topology on $G \times X$ induced by $d_{\Psi, S, k, \Lambda}$ coincides with the product topology.

\section{Strong transfer REDUCibility}

In this section we introduce the notion of strong transfer reducibility for groups which is an analogue of the notion of transfer reducibility defined in BL09, Definition 1.8].

Definition 3.1. Let $\mathcal{F}$ be a family of subgroups of $G$. The group $G$ is called strongly transfer reducible over $\mathcal{F}$ if there exists a natural number $N \in \mathbb{N}$ with the following property: For every finite symmetric subset $S \subseteq G$ containing the trivial element $e \in G$ and every natural numbers $k, n \in \mathbb{N}$ there are

- a compact contractible controlled $N$-dominated metric space $X$,

- a strong homotopy $G$-action $\Psi$ on $X$ and

- a cover $\mathcal{U}$ of $G \times X$ by open sets 
such that

(1) $\mathcal{U}$ is an open $\mathcal{F}$-cover,

(2) $\operatorname{dim}(\mathcal{U}) \leq N$

(3) for every $(g, x) \in G \times X$ there exists $U \in \mathcal{U}$ with $S_{\Psi, S, k}^{n}(g, x) \subseteq U$.

Example 3.2. Hyperbolic groups are strongly transfer reducible over the family of virtually cyclic subgroups. As metric space $X$ we choose the compactification of the Rips complex. The strong homotopy action $\Psi$ on $X$ is given by the action of the hyperbolic group on $X: \Psi\left(g_{j}, t_{j}, \ldots, g_{0}, x\right):=g_{j} \cdot \ldots \cdot g_{0} \cdot x$. For more details we refer to the proof of [BL09, Proposition 2.1].

Remark 3.3. Let $G$ be a group which is strongly transfer reducible over a family of subgroups $\mathcal{F}$. Let $H<G$ be a subgroup. We set $\mathcal{F}_{H}:=\{F \cap H \mid F \in \mathcal{F}\}$. By restricting the strong homotopy $G$-action $\Psi$ on $X$ we obtain a strong homotopy $H$-action. Moreover, we can restrict the cover $\mathcal{U}$ of $G \times X$ to a cover of $H \times X$. We conclude that $H$ is strongly transfer reducible over $\mathcal{F}_{H}$.

Following BL10, we obtain

Theorem 3.4. Every CAT(0)-group is strongly transfer reducible over the family of virtually cyclic subgroups.

Proof. Let $Y$ be a finite-dimensional CAT(0)-space on which $G$ acts cocompactly and properly. Fix a base point $x_{0} \in Y$. As metric space $X$ we will choose a (large) closed ball $\bar{B}_{R}\left(x_{0}\right) \subseteq Y$ around the base point. By BL10, Lemma 6.2$] \bar{B}_{R}\left(x_{0}\right)$ is a compact contractible controlled $(2 \cdot \operatorname{dim}(Y)+1)$-dominated metric space.

For $R>0$ we define a strong homotopy action

$$
\Psi^{R}: \coprod_{j=0}^{\infty}\left((G \times[0,1])^{j} \times G \times \bar{B}_{R}\left(x_{0}\right)\right) \rightarrow \bar{B}_{R}\left(x_{0}\right)
$$

as follows. A continuous map $c: \mathbb{R} \rightarrow Y$ is called a generalized geodesic if there are $c_{-}, c_{+} \in \mathbb{R} \cup\{ \pm \infty\}$ with $\infty \neq c_{-} \leq c_{+} \neq-\infty$ such that $c$ restricts to an isometry on the interval $\left(c_{-}, c_{+}\right)$and is locally constant on the complement of this interval (see BL10, Definition 1.1]). For $x, y \in Y$ we denote by $c_{x, y}$ the generalized geodesic satisfying $\left(c_{x, y}\right)_{-}=0, c_{x, y}(-\infty)=x$ and $c_{x, y}(\infty)=y$. For $R>0$ we consider the deformation retraction $H^{R}: Y \times[0,1] \rightarrow Y$ on the ball $\bar{B}_{R}\left(x_{0}\right)$ by projecting along geodesics, i.e.

$$
H^{R}(x, t):=c_{x, x_{0}}\left(\left(d_{Y}\left(x, x_{0}\right)-R\right) \cdot(1-t)\right) .
$$

Notice that $H_{t}^{R} \circ H_{t^{\prime}}^{R}=H_{t \cdot t^{\prime}}^{R}$. We define $\Psi^{R}$ as the associated strong homotopy action (see Remark 2.2).

For the construction of the cover $\mathcal{U}$ we have to introduce the flow space $F S(Y)$ which is the $G$-space consisting of all generalized geodesics $c: \mathbb{R} \rightarrow Y$. The metric on $F S(Y)$ is given by

$$
d_{F S(Y)}(c, d):=\int_{-\infty}^{\infty} \frac{d_{Y}(c(t), d(t))}{2 \cdot e^{|t|}} d t .
$$

We define a $G$-equivariant flow $\Phi: F S(Y) \times \mathbb{R} \rightarrow F S(Y)$ by $\Phi_{\tau}(c)(t):=c(t+\tau)$ (see [BL10, Definition 1.2]). 
By [BL10, Theorem 5.7] and [BL10, subsection 6.3] there is $\widehat{N} \in \mathbb{N}$ such that for every $\alpha>0$ there exists an open $\mathcal{V} \mathcal{C} y c$-cover $\mathcal{V}$ of $F S(Y)$ of dimension at most $\widehat{N}$ and $\epsilon>0$ such that

- $\mathcal{V} / G$ is finite and

- for every $z \in F S(Y)$ there is $V \in \mathcal{V}$ with $B_{\epsilon}\left(\Phi_{[-\alpha, \alpha]}(z)\right) \subseteq V$.

We set $N:=\max \{\widehat{N}, 2 \cdot \operatorname{dim}(Y)+1\}$.

The construction of the cover $\mathcal{U}$ is based on the contracting property described in Lemma 3.5 below. We fix $\alpha>0$ as in the assertion of Lemma 3.5. Let $\mathcal{V}$ be an open $\mathcal{V} \mathcal{C} y c$-cover of $F S(Y)$ of dimension at most $\widehat{N}$ and let $\epsilon$ be a positive real number such that the two properties mentioned above are satisfied. For this $\epsilon>0$ we obtain $R, T>0$ from Lemma 3.5. Then the cover

$$
\mathcal{U}:=\left\{\left(\Phi_{T} \circ \iota\right)^{-1}(V) \cap G \times \bar{B}_{R}\left(x_{0}\right) \mid V \in \mathcal{V}\right\}
$$

with $\iota: G \times \bar{B}_{R}\left(x_{0}\right) \rightarrow F S(Y),(g, y) \mapsto c_{g x_{0}, g y}$ has the desired properties.

In the proof of Theorem 3.4 we used the following lemma which is a modification of [BL10, Proposition 3.8], resp. [BL10, Lemma 5.12].

Lemma 3.5. Let $S \subseteq G$ be a finite symmetric subset containing the trivial element $e \in G$. Let $k, n \in \mathbb{N}$. Then there exists $\alpha>0$ with the following property: For all $\epsilon>0$ there are $R, T>0$ such that for every $(g, x) \in G \times \bar{B}_{R}\left(x_{0}\right)$ and $(h, y) \in$ $S_{\Psi^{R}, S, k}^{n}(g, x)$ there is $\tau \in[-\alpha, \alpha]$ with

$$
d_{F S(Y)}\left(\Phi_{T} \circ \iota(g, x), \Phi_{T+\tau} \circ \iota(h, y)\right) \leq \epsilon .
$$

(We use the same notation as in the proof of Theorem 3.4.)

Proof. We set $\alpha:=2 n \cdot(k+1) \cdot \alpha^{\prime}$ with $\alpha^{\prime}:=\max \left\{d_{Y}\left(g x_{0}, h x_{0}\right) \mid g, h \in S^{k+1}\right\}$. Let $\epsilon>0$. By [BL10, Proposition 3.5] there exist $R, T>0$ such that for $x, x^{\prime}, x^{\prime \prime} \in Y$ with $d_{Y}\left(x^{\prime}, x^{\prime \prime}\right) \leq \alpha^{\prime}$ and $x \in \bar{B}_{R+\alpha}\left(x^{\prime}\right)$ there is $\tau \in\left[-\alpha^{\prime}, \alpha^{\prime}\right]$ such that

$$
d_{F S(Y)}\left(\Phi_{T}\left(c_{x^{\prime}, c_{x^{\prime}, x}\left(d_{Y}\left(x^{\prime}, x\right)-R\right)}\right), \Phi_{T+\tau}\left(c_{x^{\prime \prime}, c_{x^{\prime \prime}, x}\left(d_{Y}\left(x^{\prime \prime}, x\right)-R\right)}\right)\right) \leq \frac{\epsilon}{2 n \cdot(k+1) \cdot e^{2 \alpha}} .
$$

We fix such positive real numbers $R, T$.

Next we show that for every $z \in \bar{B}_{R}\left(x_{0}\right), a \in G$ and $f \in F_{a}\left(\Psi^{R}, S, k\right)$ there is $\tau \in\left[-(k+1) \cdot \alpha^{\prime},(k+1) \cdot \alpha^{\prime}\right]$ satisfying

$$
d_{F S(Y)}\left(\Phi_{T} \circ \iota(e, z), \Phi_{T+\tau} \circ \iota\left(a^{-1}, f(z)\right)\right) \leq \frac{\epsilon}{2 n \cdot e^{\alpha}} .
$$

There are $g_{0}, \ldots, g_{k} \in S$ and $t_{1}, \ldots, t_{k} \in[0,1]$ such that $f(z)=\Psi^{R}\left(g_{k}, t_{k}, \ldots, g_{0}, z\right)$. We set $y_{0}:=z$ and $z_{0}:=z$. Moreover, we define $x_{i}:=\left(g_{i-1} \ldots g_{0}\right)^{-1} \cdot x_{0}$, $y_{i}:=\left(g_{i-1} \ldots g_{0}\right)^{-1} \cdot \Psi^{R}\left(g_{i-1}, \ldots, g_{0}, z\right)$ and $z_{i}:=\left(g_{i-1} \ldots g_{0}\right)^{-1} \cdot \Omega^{R}\left(g_{i-1}, \ldots, g_{0}, z\right)$ for $i=1, \ldots, k+1$. Notice that $y_{i}$ and $z_{i+1}$ lie on the geodesic between $x_{i}$ and $z_{i}$. We conclude $y_{i}=c_{x_{i}, z_{i+1}}\left(d_{Y}\left(x_{i}, z_{i+1}\right)-R\right)$ and $y_{i+1}=c_{x_{i+1}, z_{i+1}}\left(d_{Y}\left(x_{i+1}, z_{i+1}\right)-R\right)$. We apply the inequality (3.1) and obtain $\tau_{i} \in\left[-\alpha^{\prime}, \alpha^{\prime}\right](i=0, \ldots, k)$ with

$$
d_{F S(Y)}\left(\Phi_{T}\left(c_{x_{i}, y_{i}}\right), \Phi_{T+\tau_{i}}\left(c_{x_{i+1}, y_{i+1}}\right)\right) \leq \frac{\epsilon}{2 n \cdot(k+1) \cdot e^{2 \alpha}} .
$$


We set $s_{i}:=\sum_{l=0}^{i-1} \tau_{l} \leq(k+1) \cdot \alpha^{\prime}$ for $i=0, \ldots, k+1$. Using [BL10, Lemma 1.3] we calculate

$$
\begin{aligned}
& d_{F S(Y)}\left(\Phi_{T} \circ \iota(e, z), \Phi_{T+s_{k+1}} \circ \iota\left(a^{-1}, f(z)\right)\right) \\
& =d_{F S(Y)}\left(\Phi_{T+s_{0}}\left(c_{x_{0}, y_{0}}\right), \Phi_{T+s_{k+1}}\left(c_{x_{k+1}, y_{k+1}}\right)\right) \\
& \leq \sum_{i=0}^{k} d_{F S(Y)}\left(\Phi_{T+s_{i}}\left(c_{x_{i}, y_{i}}\right), \Phi_{T+s_{i+1}}\left(c_{x_{i+1}, y_{i+1}}\right)\right) \\
& \leq \sum_{i=0}^{k} e^{\left|s_{i}\right|} \cdot d_{F S(Y)}\left(\Phi_{T}\left(c_{x_{i}, y_{i}}\right), \Phi_{T+\tau_{i}}\left(c_{x_{i+1}, y_{i+1}}\right)\right) \\
& \leq \sum_{i=0}^{k} e^{\alpha} \cdot \frac{\epsilon}{2 n \cdot(k+1) \cdot e^{2 \alpha}}=\frac{\epsilon}{2 n \cdot e^{\alpha}} .
\end{aligned}
$$

Since $(h, y) \in S_{\Psi^{R}, S, k}^{n}(g, x)$, there are $c_{i} \in \bar{B}_{R}\left(x_{0}\right), a_{i}, b_{i} \in S, f_{i} \in F_{a_{i}}\left(\Psi^{R}, S, k\right)$ and $\tilde{f}_{i} \in F_{b_{i}}\left(\Psi^{R}, S, k\right)(i=1, \ldots, n)$ such that $c_{0}=x, c_{n}=y, f_{i}\left(c_{i-1}\right)=\tilde{f}_{i}\left(c_{i}\right)$ and $h=g a_{1}^{-1} b_{1} \ldots a_{n}^{-1} b_{n}$. By the inequality (3.2) there are $\tau_{i}, \tilde{\tau}_{i} \in\left[-(k+1) \alpha^{\prime},(k+1) \alpha^{\prime}\right]$ such that

$$
\begin{aligned}
d_{F S(Y)}\left(\Phi_{T} \circ \iota\left(e, c_{i-1}\right), \Phi_{T+\tau_{i}} \circ \iota\left(a_{i}^{-1}, f_{i}\left(c_{i-1}\right)\right)\right) & \leq \frac{\epsilon}{2 n \cdot e^{\alpha}}, \\
d_{F S(Y)}\left(\Phi_{T} \circ \iota\left(e, c_{i}\right), \Phi_{T+\tilde{\tau}_{i}} \circ \iota\left(b_{i}^{-1}, \tilde{f}_{i}\left(c_{i}\right)\right)\right) & \leq \frac{\epsilon}{2 n \cdot e^{\alpha}}
\end{aligned}
$$

for $i=1, \ldots, n$. We set $g_{i}:=g a_{1}^{-1} b_{1} \ldots a_{i}^{-1} b_{i}, \sigma_{i}:=\sum_{l=1}^{i} \tau_{i}-\tilde{\tau}_{i} \in[-\alpha, \alpha]$. Since $g_{i-1} a_{i}^{-1}=g_{i} b_{i}^{-1}, f_{i}\left(c_{i-1}\right)=\tilde{f}_{i}\left(c_{i}\right)$ and $T+\sigma_{i-1}+\tau_{i}=T+\sigma_{i}+\tilde{\tau}_{i}$ we conclude

$$
\begin{aligned}
& d_{F S(Y)}\left(\Phi_{T+\sigma_{i-1}} \circ \iota\left(g_{i-1}, c_{i-1}\right), \Phi_{T+\sigma_{i}} \circ \iota\left(g_{i}, c_{i}\right)\right) \\
& \leq d_{F S(Y)}\left(\Phi_{T+\sigma_{i-1}} \circ \iota\left(g_{i-1}, c_{i-1}\right), \Phi_{T+\sigma_{i-1}+\tau_{i}} \circ \iota\left(g_{i-1} a_{i}^{-1}, f_{i}\left(c_{i-1}\right)\right)\right) \\
& \quad \quad+d_{F S(Y)}\left(\Phi_{T+\sigma_{i}+\tilde{\tau}_{i}} \circ \iota\left(g_{i} b_{i}^{-1}, \tilde{f}_{i}\left(c_{i}\right)\right), \Phi_{T+\sigma_{i}} \circ \iota\left(g_{i}, c_{i}\right)\right) \\
& =d_{F S(Y)}\left(\Phi_{T+\sigma_{i-1}} \circ \iota\left(e, c_{i-1}\right), \Phi_{T+\sigma_{i-1}+\tau_{i}} \circ \iota\left(a_{i}^{-1}, f_{i}\left(c_{i-1}\right)\right)\right) \\
& \quad+d_{F S(Y)}\left(\Phi_{T+\sigma_{i}+\tilde{\tau}_{i}} \circ \iota\left(b_{i}^{-1}, \tilde{f}_{i}\left(c_{i}\right)\right), \Phi_{T+\sigma_{i}} \circ \iota\left(e, c_{i}\right)\right) \\
& \leq e^{\left|\sigma_{i-1}\right|} \cdot d_{F S(Y)}\left(\Phi_{T} \circ \iota\left(e, c_{i-1}\right), \Phi_{T+\tau_{i}} \circ \iota\left(a_{i}^{-1}, f_{i}\left(c_{i-1}\right)\right)\right) \\
& \quad+e^{\left|\sigma_{i}\right|} \cdot d_{F S(Y)}\left(\Phi_{T+\tilde{\tau}_{i}} \circ \iota\left(b_{i}^{-1}, \tilde{f}_{i}\left(c_{i}\right)\right), \Phi_{T} \circ \iota\left(e, c_{i}\right)\right) \\
& \leq e^{\alpha} \cdot \frac{\epsilon}{2 n \cdot e^{\alpha}}+e^{\alpha} \cdot \frac{\epsilon}{2 n \cdot e^{\alpha}}=\frac{\epsilon}{n} .
\end{aligned}
$$

For $\tau:=\sigma_{n}$ we obtain

$$
\begin{aligned}
& d_{F S(Y)}\left(\Phi_{T} \circ \iota(g, x), \Phi_{T+\tau} \circ \iota(h, y)\right) \\
& \leq \sum_{i=1}^{n} d_{F S(Y)}\left(\Phi_{T+\sigma_{i-1}} \circ \iota\left(g_{i-1}, c_{i-1}\right), \Phi_{T+\sigma_{i}} \circ \iota\left(g_{i}, c_{i}\right)\right) \\
& \leq \sum_{i=1}^{n} \frac{\epsilon}{n}=\epsilon .
\end{aligned}
$$

This finishes the proof of Lemma 3.5 .

The proof of Theorem 1.1 is based on the following proposition, which is a modification of [BL09, Proposition 3.9]. 
Proposition 3.6. Let $G$ be a group which is strongly transfer reducible over a family $\mathcal{F}$ of subgroups. Let $N$ be the number appearing in the definition of "strongly transfer reducible". Let $S \subseteq G$ be a finite symmetric subset containing the trivial element $e \in G$. Then for every $k \in \mathbb{N}$ there exist

- a compact contractible controlled $N$-dominated metric space $X$,

- a strong homotopy G-action $\Psi$ on $X$,

- a positive real number $\Lambda$,

- a simplicial complex $\Sigma$ of dimension $\leq N$ with a simplicial cell-preserving $G$-action and

- a G-equivariant map $f: G \times X \rightarrow \Sigma$

such that

- the isotropy groups of $\Sigma$ belong to $\mathcal{F}$ and

- $k \cdot d^{1}(f(g, x), f(h, y)) \leq d_{\Psi, S, k, \Lambda}((g, x),(h, y))$ for all $(g, x),(h, y) \in G \times X$.

Here $d^{1}$ denotes the $l^{1}$-metric on simplicial complexes; see [BLR08, subsection 4.2].

Proof. We choose a strong homotopy $G$-action $\Psi$ on a metric space $X$ and a cover $\mathcal{U}$ of $G \times X$ which satisfy the properties stated in Definition 3.1 for $S, k$ and $n:=4 N k$. Using Lemma 2.4(2) we conclude as in the proof of [BL09, Proposition 3.7] that for every $x \in X$ there exists $\Lambda_{x}>0$ and $U_{x} \in \mathcal{U}$ such that the $n$-ball around $(e, x)$ with respect to the quasi-metric $d_{\Psi, S, k, \Lambda_{x}}$ lies in $U_{x}$. Moreover, since $X$ is compact, there exists $\Lambda>0$ such that every $n$-ball with respect to the quasi-metric $d_{\Psi, S, k, \Lambda}$ lies in some $U \in \mathcal{U}$ (see the proof of [BL09, Proposition 3.7]). Let $\Sigma:=|\mathcal{U}|$ be the realization of the nerve of $\mathcal{U}$ and let $f$ be the map induced by $\mathcal{U}$, i.e.

$$
f: G \times X \rightarrow|\mathcal{U}|,(g, x) \mapsto \sum_{U \in \mathcal{U}} \frac{a_{U}(g, x)}{s(g, x)} U
$$

with $a_{U}(g, x):=\inf \left\{d_{\Psi, S, k, \Lambda}((g, x),(h, y)) \mid(h, y) \notin U\right\}$ and $s(g, x):=\sum_{U} a_{U}(g, x)$. Notice that $\left|a_{U}(g, x)-a_{U}(h, y)\right| \leq d_{\Psi, S, k, \Lambda}((g, x),(h, y))$ and hence

$$
\sum_{U \in \mathcal{U}}\left|a_{U}(g, x)-a_{U}(h, y)\right| \leq 2 N \cdot d_{\Psi, S, k, \Lambda}((g, x),(h, y))
$$

for all $(g, x),(h, y) \in G \times X$. We calculate

$$
\begin{aligned}
d^{1}(f(g, x), f(h, y)) & =\sum_{U \in \mathcal{U}}\left|\frac{a_{U}(g, x)}{s(g, x)}-\frac{a_{U}(h, y)}{s(h, y)}\right| \\
& =\sum_{U \in \mathcal{U}}\left|\frac{a_{U}(g, x)-a_{U}(h, y)}{s(g, x)}+\frac{a_{U}(h, y) \cdot(s(h, y)-s(g, x))}{s(g, x) \cdot s(h, y)}\right| \\
& \leq \frac{\sum_{U \in \mathcal{U}}\left|a_{U}(g, x)-a_{U}(h, y)\right|}{s(g, x)}+\frac{|s(h, y)-s(g, x)|}{s(g, x)} \\
& \leq 2 \cdot \frac{\sum_{U \in \mathcal{U}}\left|a_{U}(g, x)-a_{U}(h, y)\right|}{s(g, x)} \\
& \leq 4 N \cdot \frac{d_{\Psi, S, k, \Lambda}((g, x),(h, y))}{s(g, x)} \\
& \leq \frac{d_{\Psi, S, k, \Lambda}((g, x),(h, y))}{k} .
\end{aligned}
$$


For the last inequality we used the fact that the $n$-ball around $(g, x)$ lies in some $U \in \mathcal{U}$ and hence $s(g, x) \geq n$.

\section{The obstruction CATEgory}

In this section we recall the definition of the obstruction category. In the following $\mathcal{A}$ denotes a small additive category (with strictly associative direct sum) which is provided with a strict right $G$-action.

Definition 4.1. Let $X$ be a $G$-space and let $\left(Y, d_{Y}\right)$ be a metric space with an isometric $G$-action. We consider the $G$-space $G \times X \times Y \times[1, \infty)$ with the $G$ action given by $h(g, x, y, t):=(h g, h x, h y, t)$. We define the obstruction category $\mathcal{O}^{G}\left(X,\left(Y, d_{Y}\right) ; \mathcal{A}\right)$ as follows.

An object in $\mathcal{O}^{G}\left(X,\left(Y, d_{Y}\right) ; \mathcal{A}\right)$ is a collection $A=\left(A_{g, x, y, t}\right)_{(g, x, y, t) \in G \times X \times Y \times[1, \infty)}$ of objects in $\mathcal{A}$ with the following properties:

- $A$ is locally finite, i.e. for every $z_{0} \in G \times X \times Y \times[1, \infty)$ there exists an open neighborhood $U$ such that the set $\left\{z \in G \times X \times Y \times[1, \infty) \mid A_{z} \neq 0\right\} \cap U$ is finite.

- There is a compact subset $K \subseteq G \times X \times Y$ such that $A_{g, x, y, t}=0$ whenever $(g, x, y) \notin G \cdot K$.

- We have $A_{z} \cdot g=A_{g^{-1} z}$ for all $z \in G \times X \times Y \times[1, \infty)$ and $g \in G$.

A morphism $\phi: B \rightarrow A$ is a collection of morphisms $\phi_{z, z^{\prime}}: B_{z^{\prime}} \rightarrow A_{z}$ in $\mathcal{A}\left(z, z^{\prime} \in\right.$ $G \times X \times Y \times[1, \infty))$ with the following properties:

- The sets $\left\{z \in G \times X \times Y \times[1, \infty) \mid \phi_{z, z^{\prime}} \neq 0\right\}$ and $\{z \in G \times X \times Y \times$ $\left.[1, \infty) \mid \phi_{z^{\prime}, z} \neq 0\right\}$ are finite for all $z^{\prime} \in G \times X \times Y \times[1, \infty)$.

- There are $R, T>0$ and a finite subset $F \subseteq G$ such that $\phi_{(g, x, y, t),\left(g^{\prime}, x^{\prime}, y^{\prime}, t^{\prime}\right)}=$ 0 whenever $g^{-1} g^{\prime} \notin F$ or $d_{Y}\left(y, y^{\prime}\right)>R$ or $\left|t-t^{\prime}\right|>T$.

- The set

$$
\left\{\left((x, t),\left(x^{\prime}, t^{\prime}\right)\right) \in(X \times[1, \infty))^{2} \mid \exists g, g^{\prime} \in G, y, y^{\prime} \in Y: \phi_{(g, x, y, t),\left(g^{\prime}, x^{\prime}, y^{\prime}, t^{\prime}\right)} \neq 0\right\}
$$

lies in the equivariant continuous control condition $\mathcal{E}_{G c c}^{X}$ defined in BLR08, section 3.2].

- We have $\phi_{z, z^{\prime}} \cdot g=\phi_{g^{-1} z, g^{-1} z^{\prime}}$ for all $z, z^{\prime} \in G \times X \times Y \times[1, \infty)$ and $g \in G$. Composition is given by matrix multiplication, i.e.

$$
(\psi \circ \phi)_{z, z^{\prime \prime}}:=\sum_{z^{\prime} \in G \times X \times Y \times[1, \infty)} \psi_{z, z^{\prime}} \circ \psi_{z^{\prime}, z^{\prime \prime}}
$$

The obstruction category $\mathcal{O}^{G}\left(X,\left(Y, d_{Y}\right) ; \mathcal{A}\right)$ inherits the structure of an additive category from $\mathcal{A}$.

We use the same notation as in BL09, subsection 4.4] which slightly differs from the notation used in BLR08] (see [BL09, Remark 4.10]).

The construction is functorial in $Y$ : Let $f: Y \rightarrow Y^{\prime}$ be a $G$-equivariant map with the property that for every $r>0$ there exists $R>0$ such that $d_{Y^{\prime}}\left(f\left(y_{1}\right), f\left(y_{2}\right)\right)<R$ whenever $d_{Y}\left(y_{1}, y_{2}\right)<r$. Then the map $f$ induces a functor $f_{*}: \mathcal{O}^{G}(X, Y ; \mathcal{A}) \rightarrow$ $\mathcal{O}^{G}\left(X, Y^{\prime} ; \mathcal{A}\right)$ with $f_{*}(A)_{g, x, y^{\prime}, t}:=\bigoplus_{y \in f^{-1}\left(\left\{y^{\prime}\right\}\right)} A_{g, x, y, t}$.

We are mostly interested in $\mathcal{O}^{G}\left(E_{\mathcal{F}} G, \mathrm{pt} ; \mathcal{A}\right)$ because of the following proposition, which is proven in [BLR08, Proposition 3.8]. 
Proposition 4.2. Let $G$ be a group and let $m_{0} \in \mathbb{Z}$ be such that

$$
K_{m}\left(\mathcal{O}^{G}\left(E_{\mathcal{F}} G, \mathrm{pt} ; \mathcal{A}\right)\right)=0
$$

for all $m \geq m_{0}$ and all additive $G$-categories $\mathcal{A}$. Then the assembly map (1.1) is an isomorphism for all $m \in \mathbb{Z}$ and all additive $G$-categories $\mathcal{A}$.

The reason why we study the category $\mathcal{O}^{G}\left(E_{\mathcal{F}} G,\left(Y, d_{Y}\right) ; \mathcal{A}\right)$ not only for $Y:=\mathrm{pt}$ is that we need room for certain constructions. Moreover, we want to consider simultaneously metric spaces $\left(Y_{n}, d_{n}\right)$ with isometric $G$-action $(n \in \mathbb{N})$. In analogy to [BLR08, subsection 3.4] we define the additive subcategory

$$
\mathcal{O}^{G}\left(E_{\mathcal{F}} G,\left(Y_{n}, d_{n}\right)_{n \in \mathbb{N}} ; \mathcal{A}\right) \subseteq \prod_{n \in \mathbb{N}} \mathcal{O}^{G}\left(E_{\mathcal{F}} G,\left(Y_{n}, d_{n}\right) ; \mathcal{A}\right)
$$

by requiring additional conditions on the morphisms. A morphism $\phi=(\phi(n))_{n \in \mathbb{N}}$ is allowed if there are $R>0$ and a finite subset $F \subseteq G$ (not depending on $n$ ) such that $\phi(n)_{(g, x, y, t),\left(g^{\prime}, x^{\prime}, y^{\prime}, t^{\prime}\right)}=0$ whenever $g^{-1} g^{\prime} \notin F$ or $d_{n}\left(y, y^{\prime}\right)>R$.

The inclusion

$$
\bigoplus_{n \in \mathbb{N}} \mathcal{O}^{G}\left(E_{\mathcal{F}} G,\left(Y_{n}, d_{n}\right) ; \mathcal{A}\right) \rightarrow \mathcal{O}^{G}\left(E_{\mathcal{F}} G,\left(Y_{n}, d_{n}\right)_{n \in \mathbb{N}} ; \mathcal{A}\right)
$$

is a Karoubi filtration and we denote the quotient by $\mathcal{O}^{G}\left(E_{\mathcal{F}} G,\left(Y_{n}, d_{n}\right)_{n \in \mathbb{N}} ; \mathcal{A}\right)^{>\oplus}$. Notice that a sequence of $G$-equivariant maps $\left(f_{n}: Y_{n} \rightarrow Y_{n}^{\prime}\right)_{n \in \mathbb{N}}$ induces a functor $\left(f_{n}\right)_{*}: \mathcal{O}^{G}\left(E_{\mathcal{F}} G,\left(Y_{n}, d_{n}\right)_{n \in \mathbb{N}} ; \mathcal{A}\right) \rightarrow \mathcal{O}^{G}\left(E_{\mathcal{F}} G,\left(Y_{n}^{\prime}, d_{n}^{\prime}\right)_{n \in \mathbb{N}} ; \mathcal{A}\right)$ if for every $r>0$ there exists $R>0$ such that $d_{n}^{\prime}\left(f_{n}\left(y_{1}\right), f_{n}\left(y_{2}\right)\right)<R$ whenever $d_{n}\left(y_{1}, y_{2}\right)<r$.

\section{Outline of the Proof of TheOrem 1.1}

In this section we sketch the proof of Theorem 1.1 .

Since the class of groups satisfying the $K$-theoretic Farrell-Jones conjecture is closed under directed colimits, it suffices to prove the bijectivity of the $K$-theoretic assembly map (1.1) for every finitely generated subgroup $H$ of $G$ (with respect to the family $\mathcal{F}_{H}:=\{F \cap H \mid F \in \mathcal{F}\}$ ). Moreover, strong transfer reducibility is stable under taking subgroups (see Remark 3.3). This shows that it is enough to prove Theorem 1.1 for finitely generated groups. Therefore we can and will assume that $G$ is finitely generated.

We fix a finite symmetric generating subset $S \subseteq G$ which contains the trivial element $e \in G$. We apply Proposition [3.6 to $S^{n}:=\left\{s_{1} \cdot s_{2} \cdot \ldots \cdot s_{n} \mid s_{i} \in S\right\} \subseteq G$ and $k:=n$ and obtain

- compact contractible controlled $N$-dominated metric spaces $X_{n}$,

- strong homotopy $G$-actions $\Psi_{n}$ on $X_{n}$,

- positive real numbers $\Lambda_{n}$,

- simplicial complexes $\Sigma_{n}$ of dimension $\leq N$ with simplicial cell-preserving $G$-actions and

- $G$-equivariant maps $f_{n}: G \times X_{n} \rightarrow \Sigma_{n}$

such that

- the isotropy groups of $\Sigma_{n}$ belong to $\mathcal{F}$ and

- $n \cdot d^{1}\left(f_{n}(g, x), f_{n}(h, y)\right) \leq d_{\Psi_{n}, S^{n}, n, \Lambda_{n}}((g, x),(h, y))$ for all $(g, x),(h, y) \in$ $G \times X_{n}$. 
We abbreviate $d_{n}:=d_{\Psi_{n}, S^{n}, n, \Lambda_{n}}$.

By Proposition 4.2 it suffices to show that

$$
K_{m}\left(\mathcal{O}^{G}\left(E_{\mathcal{F}} G, \mathrm{pt} ; \mathcal{A}\right)\right)=0
$$

for all $m \geq 1$. Theorem 1.1 is a consequence of the following commuting diagram:

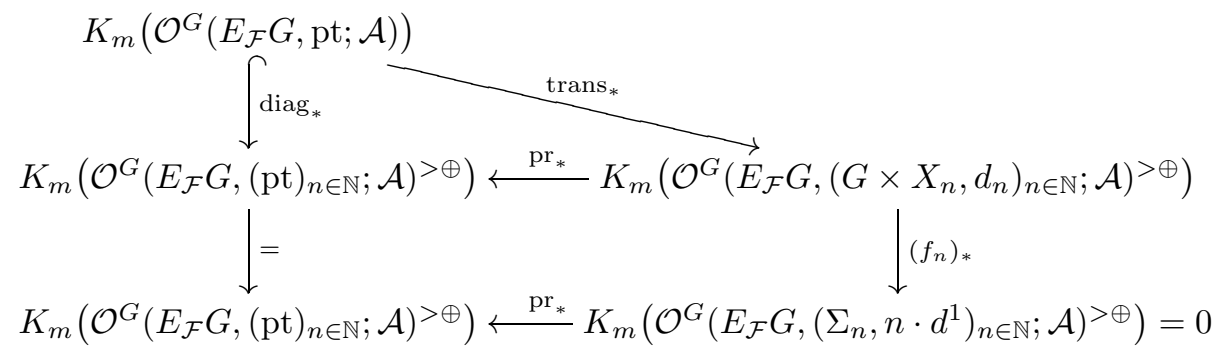

The map $\operatorname{diag}_{*}: K_{m}\left(\mathcal{O}^{G}\left(E_{\mathcal{F}} G, \mathrm{pt} ; \mathcal{A}\right)\right) \rightarrow K_{m}\left(\mathcal{O}^{G}\left(E_{\mathcal{F}} G,(\mathrm{pt})_{n \in \mathbb{N}} ; \mathcal{A}\right)^{>\oplus}\right)$ is induced by the diagonal map. The injectivity of this map can easily be shown by a diagram chase in the diagram

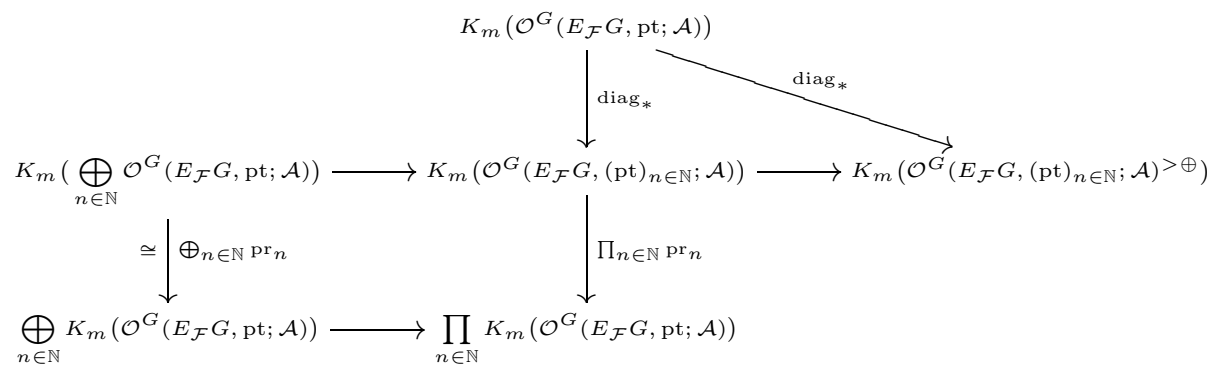

where the middle row comes from the Karoubi filtration. (Notice that the composition $\prod_{n \in \mathbb{N}} \mathrm{pr}_{n} \circ \operatorname{diag}_{*}$ in the middle column is the diagonal map.) The transfer map

$$
\operatorname{trans}_{*}: K_{m}\left(\mathcal{O}^{G}\left(E_{\mathcal{F}} G, \mathrm{pt} ; \mathcal{A}\right)\right) \rightarrow K_{m}\left(\mathcal{O}^{G}\left(E_{\mathcal{F}} G,\left(G \times X_{n}, d_{n}\right)_{n \in \mathbb{N}} ; \mathcal{A}\right)^{>\oplus}\right)
$$

will be constructed in section 7 . The maps $\operatorname{pr}_{*}$ are induced by the projections pr: $G \times X_{n} \rightarrow$ pt, resp. pr: $\Sigma_{n} \rightarrow$ pt. The equation

$$
K_{m}\left(\mathcal{O}^{G}\left(E_{\mathcal{F}} G,\left(\Sigma_{n}, n \cdot d^{1}\right)_{n \in \mathbb{N}} ; \mathcal{A}\right)^{>\oplus}\right)=0
$$

is proved in BLR08, Theorem 7.2].

\section{Preparations for the transfer}

We will define the transfer map

$$
\operatorname{trans}_{*}: K_{m}\left(\mathcal{O}^{G}\left(E_{\mathcal{F}} G, \mathrm{pt} ; \mathcal{A}\right)\right) \rightarrow K_{m}\left(\mathcal{O}^{G}\left(E_{\mathcal{F}} G,\left(G \times X_{n}, d_{n}\right)_{n \in \mathbb{N}} ; \mathcal{A}\right)^{>\oplus}\right)
$$

as the map induced by a functor

$$
\text { trans: } \mathcal{O}^{G}\left(E_{\mathcal{F}} G, \mathrm{pt} ; \mathcal{A}\right) \rightarrow \widetilde{\operatorname{ch}}_{\mathrm{hfd}} \mathcal{O}^{G}\left(E_{\mathcal{F}} G,\left(G \times X_{n}, d_{n}\right)_{n \in \mathbb{N}} ; \mathcal{A}\right)^{>\oplus} \text {. }
$$

In this section we give a quite short review of the construction of the category $\widetilde{c h}_{\mathrm{hfd}} \mathcal{O}^{G}\left(E_{\mathcal{F}} G,\left(G \times X_{n}, d_{n}\right)_{n \in \mathbb{N}} ; \mathcal{A}\right)^{>\oplus}$. For more details we refer to [BLR08, subsection 6.2]. 
For a metric space $\left(Y, d_{Y}\right)$ with an isometric $G$-action we define the category $\overline{\mathcal{O}}^{G}\left(E_{\mathcal{F}} G,\left(Y, d_{Y}\right) ; \mathcal{A}^{\kappa}\right)$ in the same way as in section 4 , but we replace $\mathcal{A}$ by $\mathcal{A}^{\kappa}$ for a fixed (suitably chosen) infinite cardinal $\kappa$ and drop the assumption that the support of objects is locally finite. Moreover, instead of requiring for a morphism $\phi=\left(\phi_{z, z^{\prime}}\right)_{z, z^{\prime} \in G \times E_{\mathcal{F}} G \times Y \times[1, \infty)}$ that the sets $\left\{z \mid \phi_{z, z^{\prime}} \neq 0\right\}$ and $\left\{z \mid \phi_{z^{\prime}, z} \neq 0\right\}$ are finite, we define a morphism

$$
\phi=\left(\phi_{z, z^{\prime}}\right): B=\left(B_{z^{\prime}}\right)_{z^{\prime} \in G \times E_{\mathcal{F}} G \times Y \times[1, \infty)} \rightarrow A=\left(A_{z}\right)_{z \in G \times E_{\mathcal{F}} G \times Y \times[1, \infty)}
$$

to be a morphism $\bigoplus_{z^{\prime} \in G \times E_{\mathcal{F}} G \times Y \times[1, \infty)} B_{z^{\prime}} \rightarrow \bigoplus_{z \in G \times E_{\mathcal{F}} G \times Y \times[1, \infty)} A_{z}$ in the category $\mathcal{A}^{\kappa}$. For a sequence $\left(Y_{n}, d_{n}\right)_{n \in \mathbb{N}}$ of metric spaces with isometric $G$-action we define

$$
\overline{\mathcal{O}}^{G}\left(E_{\mathcal{F}} G,\left(Y_{n}, d_{n}\right)_{n \in \mathbb{N}} ; \mathcal{A}^{\kappa}\right) \subset \prod_{n \in \mathbb{N}} \overline{\mathcal{O}}^{G}\left(E_{\mathcal{F}} G,\left(Y_{n}, d_{n}\right) ; \mathcal{A}^{\kappa}\right)
$$

by requiring additional conditions on the morphisms precisely as in section 4. The inclusion

$$
\bigoplus_{n \in \mathbb{N}} \overline{\mathcal{O}}^{G}\left(E_{\mathcal{F}} G,\left(Y_{n}, d_{n}\right) ; \mathcal{A}^{\kappa}\right) \rightarrow \overline{\mathcal{O}}^{G}\left(E_{\mathcal{F}} G,\left(Y_{n}, d_{n}\right)_{n \in \mathbb{N}} ; \mathcal{A}^{\kappa}\right)
$$

is a Karoubi filtration and we denote the quotient by $\overline{\mathcal{O}}^{G}\left(E_{\mathcal{F}} G,\left(Y_{n}, d_{n}\right)_{n \in \mathbb{N}} ; \mathcal{A}^{\kappa}\right)^{>\oplus}$. For the rest of this section we abbreviate

$$
\begin{aligned}
\mathcal{O} & :=\mathcal{O}^{G}\left(E_{\mathcal{F}} G,\left(Y_{n}, d_{n}\right)_{n \in \mathbb{N}} ; \mathcal{A}\right)^{>\oplus} \\
\overline{\mathcal{O}} & :=\overline{\mathcal{O}}^{G}\left(E_{\mathcal{F}} G,\left(Y_{n}, d_{n}\right)_{n \in \mathbb{N}} ; \mathcal{A}^{\kappa}\right)^{>\oplus} .
\end{aligned}
$$

One should think of the inclusion $\mathcal{O} \subset \overline{\mathcal{O}}$ as an inclusion of a full additive subcategory on objects satisfying finiteness conditions into a large category which gives room for constructions.

Let $\mathcal{C}$ be an additive category (e.g. $\mathcal{O}$ or $\overline{\mathcal{O}})$. We write $\operatorname{Idem}(\mathcal{C})$ for its idempotent completion. We define $\operatorname{ch}_{\mathrm{f}}(\mathcal{C})$ to be the category of chain complexes in $\mathcal{C}$ that are bounded above and below and $\mathrm{ch}^{\geq}(\mathcal{C})$ to be the category of chain complexes that are bounded below. We write $\operatorname{ch}_{\mathrm{hf}}(\operatorname{Idem}(\mathcal{O}) \subset \operatorname{Idem}(\overline{\mathcal{O}}))$ for the full subcategory of $\mathrm{ch}^{\geq}(\operatorname{Idem}(\overline{\mathcal{O}}))$ consisting of chain complexes which are chain homotopy equivalent to a chain complex in $\operatorname{ch}_{\mathrm{f}}(\operatorname{Idem}(\mathcal{O}))$. We write $\operatorname{ch}_{\mathrm{hfd}}(\mathcal{O})$ for the full subcategory of $\mathrm{ch}^{\geq} \operatorname{Idem}(\overline{\mathcal{O}})$ consisting of objects $C$ which are homotopy retracts of objects in $\operatorname{ch}_{\mathrm{f}}(\mathcal{O})$; i.e. there exists a diagram $C \stackrel{i}{\rightarrow} D \stackrel{r}{\rightarrow} C$ with $D \in \operatorname{ch}_{\mathrm{f}}(\mathcal{O})$ such that the composition $r \circ i$ is chain homotopic to the identity on $C$.

The category $\operatorname{ch}_{\mathrm{hfd}}(\mathcal{O})$ is a Waldhausen category: The notion of chain homotopy leads to a notion of weak equivalence, and we define cofibrations to be those chain maps which are degreewise the inclusion of a direct summand. The following lemma is proven in [BLR08, Lemma 6.5].

Lemma 6.1. The inclusion $\mathcal{O} \subset \operatorname{ch}_{\mathrm{hfd}}(\mathcal{O})$ induces an equivalence on $K_{m}$ for all $m \geq 1$.

We recall from BR05, subsection 8.2] that for a given Waldhausen category $\mathcal{W}$ there exists a Waldhausen category $\widetilde{\mathcal{W}}$ whose objects are sequences

$$
C_{0} \stackrel{c_{0}}{\rightarrow} C_{1} \stackrel{c_{1}}{\rightarrow} C_{2} \stackrel{c_{2}}{\rightarrow} \cdots
$$


where the $c_{\alpha}$ are morphisms in $\mathcal{W}$ that are both cofibrations and weak equivalences. A morphism $f$ in $\widetilde{\mathcal{W}}$ is represented by a sequence of morphisms $\left(f_{\alpha}, f_{\alpha+1}, f_{\alpha+2}, \cdots\right)$ which makes the diagram

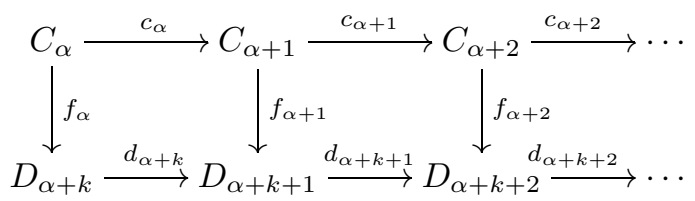

$\left(\alpha, k \in \mathbb{N}_{0}\right)$ commutative. If we enlarge $\alpha$ or $k$, the resulting diagrams represent the same morphism; i.e. we identify $\left(f_{\alpha}, f_{\alpha+1}, f_{\alpha+2}, \cdots\right)$ with $\left(f_{\alpha+1}, f_{\alpha+2}, f_{\alpha+3}, \cdots\right)$ but also with $\left(d_{\alpha+k} \circ f_{\alpha}, d_{\alpha+k+1} \circ f_{\alpha+1}, d_{\alpha+k+2} \circ f_{\alpha+2}, \cdots\right)$. Sending an object to the constant sequence defines an inclusion $\mathcal{W} \rightarrow \widetilde{\mathcal{W}}$. According to BR05, Proposition 8.2] the inclusion induces an isomorphism on $K_{m}$ for $m \geq 0$ under some mild conditions for $\mathcal{W}$. These conditions will be satisfied in all our examples.

\section{THE TRANSFER}

In this section we define a functor

$$
\text { trans: } \mathcal{O}^{G}\left(E_{\mathcal{F}} G, \mathrm{pt} ; \mathcal{A}\right) \rightarrow \widetilde{\mathrm{ch}}_{\mathrm{hfd}}\left(\mathcal{O}^{G}\left(E_{\mathcal{F}} G,\left(G \times X_{n}, d_{n}\right)_{n \in \mathbb{N}} ; \mathcal{A}\right)^{>\oplus}\right)
$$

which induces the desired transfer map

$$
\operatorname{trans}_{*}: K_{m}\left(\mathcal{O}^{G}\left(E_{\mathcal{F}} G, \mathrm{pt} ; \mathcal{A}\right)\right) \rightarrow K_{m}\left(\mathcal{O}^{G}\left(E_{\mathcal{F}} G,\left(G \times X_{n}, d_{n}\right)_{n \in \mathbb{N}} ; \mathcal{A}\right)^{>\oplus}\right) .
$$

The strong homotopy $G$-actions $\Psi_{n}$ on $X_{n}$ induce $G$-spaces $M_{n}$ (see Remark 2.2). Moreover, we obtain filtrations

$$
M_{n}^{0} \subseteq M_{n}^{1} \subseteq M_{n}^{2} \subseteq \cdots \subseteq \bigcup_{\alpha \in \mathbb{N}_{0}} M_{n}^{\alpha}=M_{n}
$$

with

$$
M_{n}^{\alpha}:=\left\{\Psi_{n}\left(?, t_{\alpha}, g_{\alpha-1}, \ldots, g_{0}, x\right) \mid t_{i} \in[0,1], g_{i} \in S^{\alpha}, x \in X_{n}\right\} .
$$

We define maps $i_{n}^{\alpha}: M_{n}^{\alpha} \rightarrow X_{n}$ and $p_{n}: X_{n} \rightarrow M_{n}^{0} \subseteq M_{n}^{\alpha}$ by $i_{n}^{\alpha}(f):=f(e)$ and $p_{n}(x):=\Psi_{n}(?, x)$. Also, $H_{n}^{\alpha}: M_{n}^{\alpha} \times[0,1] \rightarrow M_{n}^{\alpha},(f, t) \mapsto f(?, t, e)$ is a homotopy between $H_{n}^{\alpha}(?, 0)=p_{n} \circ i_{n}^{\alpha}$ and $H_{n}^{\alpha}(?, 1)=\mathrm{id}$.

We denote by $C_{*}(n, \alpha) \subseteq C_{*}^{\text {sing }}\left(G \times X_{n}\right)$, resp. $D_{*}(n, \alpha) \subseteq C_{*}^{\text {sing }}\left(G \times M_{n}^{\alpha}\right)$, the chain subcomplex generated by all singular simplices $\sigma: \Delta \rightarrow G \times X_{n}$, resp. $G \times M_{n}^{\alpha}$, for which the diameter of $\sigma(\Delta)$, resp. (id $\left.\times i_{n}^{\alpha}\right) \circ \sigma(\Delta)$, is less than or equal to $2 \alpha$. Here $C_{*}(n, \alpha)$ and $D_{*}(n, \alpha)$ are both chain complexes over $G \times X_{n}$ via the barycenter map, resp. the composition of the barycenter map with id $\times i_{n}^{\alpha}$.

Let $A$ be an object in $\mathcal{O}^{G}\left(E_{\mathcal{F}} G\right.$, pt; $\left.\mathcal{A}\right)$. We define objects $\left(A \otimes C_{*}(n, \alpha)\right)_{n \in \mathbb{N}}$ and $\left(A \otimes D_{*}(n, \alpha)\right)_{n \in \mathbb{N}}$ in $\mathrm{ch}^{\geq} \overline{\mathcal{O}}^{G}\left(E_{\mathcal{F}} G,\left(G \times X_{n}, d_{n}\right)_{n \in \mathbb{N}} ; \mathcal{A}^{\kappa}\right)$ by

$$
\begin{aligned}
& \left(A \otimes C_{k}(n, \alpha)\right)_{(g, e,(h, x), t)}:= \begin{cases}A_{(g, e, t)} \otimes C_{k}(n, \alpha)_{(g, x)} & \text { if } g=h, \\
0 & \text { otherwise; }\end{cases} \\
& \left(A \otimes D_{k}(n, \alpha)\right)_{(g, e,(h, x), t)}:= \begin{cases}A_{(g, e, t)} \otimes D_{k}(n, \alpha)_{(g, x)} & \text { if } g=h, \\
0 & \text { otherwise. }\end{cases}
\end{aligned}
$$

The differentials are given by id $\otimes \partial$.

Furthermore, BL09, Lemma 8.4] implies that $\left(A \otimes C_{*}(n, \alpha)\right)_{n \in \mathbb{N}}$ is an object in $\operatorname{ch}_{\mathrm{hfd}}\left(\mathcal{O}^{G}\left(E_{\mathcal{F}} G,\left(G \times X_{n}, d_{n}\right)_{n \in \mathbb{N}} ; \mathcal{A}\right)^{>\oplus}\right)$. The usual construction of the chain homotopy associated to the homotopy id $\times H_{n}^{\alpha}: G \times M_{n}^{\alpha} \rightarrow G \times M_{n}^{\alpha}$ yields a chain 
homotopy between $\left(\mathrm{id} \times\left(p_{n} \circ i_{n}^{\alpha}\right)\right)_{*}: C_{*}(n, \alpha) \rightarrow C_{*}(n, \alpha)$ and the identity. Let $\sigma: \Delta \rightarrow G \times M_{n}^{\alpha}$ be a singular simplex for which the diameter of $i_{n}^{\alpha} \circ \operatorname{pr}_{M_{n}^{\alpha}} \circ \sigma(\Delta)$ is less than or equal to $2 \alpha$. Since $i_{n}^{\alpha} \circ H_{n}^{\alpha}(?, t)=i_{n}^{\alpha}$ for all $t$, the diameter of images of simplices in $\Delta \times[0,1]$ under $i_{n}^{\alpha} \circ \operatorname{pr}_{M_{n}^{\alpha}} \circ\left(\mathrm{id} \times H_{n}^{\alpha}\right) \circ(\sigma \times \mathrm{id})$ is again bounded by $2 \alpha$. This shows that $\left(A \otimes D_{*}(n, \alpha)\right)_{n \in \mathbb{N}}$ is a homotopy retract of $\left(A \otimes C_{*}(n, \alpha)\right)_{n \in \mathbb{N}}$ and that $\left(A \otimes D_{*}(n, \alpha)\right)_{n \in \mathbb{N}}$ is an object in $\operatorname{ch}_{\mathrm{hfd}}\left(\mathcal{O}^{G}\left(E_{\mathcal{F}} G,\left(G \times X_{n}, d_{n}\right)_{n \in \mathbb{N}} ; \mathcal{A}\right)^{>\oplus}\right)$, too.

We define $\operatorname{trans}(A)$ as the object

$\left(A \otimes D_{*}(n, 0)\right)_{n \in \mathbb{N}} \stackrel{\mathrm{id} \otimes \mathrm{inc}}{\longrightarrow}\left(A \otimes D_{*}(n, 1)\right)_{n \in \mathbb{N}} \stackrel{\mathrm{id} \otimes \mathrm{inc}}{\longrightarrow}\left(A \otimes D_{*}(n, 2)\right)_{n \in \mathbb{N}} \stackrel{\mathrm{id} \otimes \mathrm{inc}}{\longrightarrow} \ldots$

in $\widetilde{\mathrm{ch}}_{\mathrm{hfd}} \mathcal{O}^{G}\left(E_{\mathcal{F}} G,\left(G \times X_{n}, d_{n}\right)_{n \in \mathbb{N}} ; \mathcal{A}\right)^{>\oplus}$.

Let $\phi: A \rightarrow B$ be a morphism in $\mathcal{O}^{G}\left(E_{\mathcal{F}} G, \mathrm{pt} ; \mathcal{A}\right)$. We choose $\alpha_{0} \in \mathbb{N}$ such that $\phi_{(g, e, t),\left(g^{\prime}, e^{\prime}, t^{\prime}\right)}=0$ whenever $g^{-1} g^{\prime} \notin S^{\alpha_{0}}$. For $\alpha \geq \alpha_{0}$ we define

$$
(\phi \otimes m(n, \alpha))_{n \in \mathbb{N}}:\left(A \otimes D_{*}(n, \alpha)\right)_{n \in \mathbb{N}} \rightarrow\left(B \otimes D_{*}(n, \alpha+1)\right)_{n \in \mathbb{N}},
$$

whose components are given by

$$
(\phi \otimes m(n, \alpha))_{(g, e,(g, x), t),\left(g^{\prime}, e^{\prime},\left(g^{\prime}, x^{\prime}\right), t^{\prime}\right)}=\phi_{(g, e, t),\left(g^{\prime}, e^{\prime}, t^{\prime}\right)} \otimes m_{g^{-1} g^{\prime}}(n, \alpha)_{(g, x),\left(g^{\prime}, x^{\prime}\right)} .
$$

Here, $m_{h}(n, \alpha): D_{*}(n, \alpha) \rightarrow D_{*}(n, \alpha+1)\left(h \in S^{\alpha_{0}} \subseteq S^{\alpha}\right)$ is the map induced by

$$
G \times M_{n}^{\alpha} \rightarrow G \times M_{n}^{\alpha+1},(g, f) \mapsto\left(g h^{-1}, c_{h}(f)\right)
$$

where $c_{h}: M_{n}^{\alpha} \rightarrow M_{n}^{\alpha+1}, f \mapsto f(?, 1, h)$ is the restriction of the $G$-action on $M_{n}$.

Then $(\phi \otimes m(n, \alpha))_{n \in \mathbb{N}}$ is a morphism in $\operatorname{ch}_{\mathrm{hfd}} \mathcal{O}^{G}\left(E_{\mathcal{F}} G,\left(G \times X_{n}, d_{n}\right)_{n \in \mathbb{N}} ; \mathcal{A}\right)^{>\oplus}$. The crucial point is that we have

$$
(\phi \otimes m(n, \alpha))_{(g, e,(g, x), t),\left(g^{\prime}, e^{\prime},\left(g^{\prime}, x^{\prime}\right), t^{\prime}\right)}=0
$$

for $n \geq \alpha \geq \alpha_{0}$ whenever $d_{n}\left((g, x),\left(g^{\prime}, x^{\prime}\right)\right)>2$. We will prove this fact. Suppose that $(\phi \otimes m(n, \alpha))_{(g, e,(g, x), t),\left(g^{\prime}, e^{\prime},\left(g^{\prime}, x^{\prime}\right), t^{\prime}\right)} \neq 0$ with $n \geq \alpha \geq \alpha_{0}$. We want to show $d_{n}\left((g, x),\left(g^{\prime}, x^{\prime}\right)\right) \leq 2$. We have $\phi_{(g, e, t),\left(g^{\prime}, e^{\prime}, t^{\prime}\right)} \neq 0$ and $m_{g^{-1} g^{\prime}}(n, \alpha)_{(g, x),\left(g^{\prime}, x^{\prime}\right)} \neq 0$. The first inequality shows $g^{-1} g^{\prime} \in S^{\alpha_{0}} \subseteq S^{\alpha}$. The second inequality implies the existence of an element $f \in M_{n}^{\alpha}$ such that $f(e)=x^{\prime}$ and $c_{g^{-1} g^{\prime}}(f)(e)=x$. We write $f=\Psi_{n}\left(?, t_{\alpha}, g_{\alpha-1}, \ldots, g_{0}, y\right)$ with $t_{i} \in[0,1], g_{i} \in S^{\alpha}, y \in X_{n}$. We obtain

$$
\left(g^{\prime}, x^{\prime}\right)=\left(g^{\prime}, f(e)\right),(g, x)=\left(g, c_{g^{-1}} g^{\prime}(f)(e)\right) \in S_{\Psi_{n}, S^{\alpha}, \alpha}^{1}\left(g^{\prime} k, y\right)
$$

with $k:=g_{\alpha-1} \cdot \ldots \cdot g_{0}$. This implies

$$
d_{n}\left((g, x),\left(g^{\prime}, x^{\prime}\right)\right) \leq d_{n}\left((g, x),\left(g^{\prime} k, y\right)\right)+d_{n}\left(\left(g^{\prime}, x^{\prime}\right),\left(g^{\prime} k, y\right)\right) \leq 2 .
$$

Finally, we obtain a functor

$$
\text { trans: } \mathcal{O}^{G}\left(E_{\mathcal{F}} G, \mathrm{pt} ; \mathcal{A}\right) \rightarrow \widetilde{\operatorname{ch}}_{\mathrm{hfd}} \mathcal{O}^{G}\left(E_{\mathcal{F}} G,\left(G \times X_{n}, d_{n}\right)_{n \in \mathbb{N}} ; \mathcal{A}\right)^{>\oplus}
$$

which sends a morphism $\phi: A \rightarrow B$ to the morphism represented by

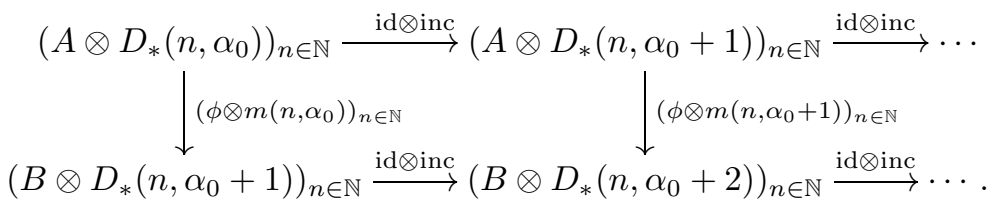

See [BLR08, Lemma 6.16] for the proof that

$\operatorname{pr}_{*} \circ \operatorname{trans}_{*}=\operatorname{diag}_{*}: K_{m}\left(\mathcal{O}^{G}\left(E_{\mathcal{F}} G, \mathrm{pt} ; \mathcal{A}\right)\right) \rightarrow K_{m}\left(\mathcal{O}^{G}\left(E_{\mathcal{F}} G,(\mathrm{pt})_{n \in \mathbb{N}} ; \mathcal{A}\right)^{>\oplus}\right)$. 


\section{REFERENCES}

[BFJR04] Bartels, Arthur; Farrell, Tom; Jones, Lowell; Reich, Holger: On the isomorphism conjecture in algebraic $K$-theory. Topology 43 (2004), no. 1, 157-213. MR2030590 (2004m:19004)

[BL09] Bartels, Arthur; Lück, Wolfgang: The Borel Conjecture for hyperbolic and CAT(0)groups. arXiv:0901.0442v2.

[BL10] Bartels, Arthur; Lück, Wolfgang: Geodesic flow for CAT(0)-groups. arXiv:1003.4630v1.

[BLR08] Bartels, Arthur; Lück, Wolfgang; Reich, Holger: The $K$-theoretic Farrell-Jones conjecture for hyperbolic groups. Invent. Math. 172 (2008), no. 1, 29-70. MR2385666 (2009c:19002)

[BR05] Bartels, Arthur; Reich, Holger: On the Farrell-Jones conjecture for higher algebraic K-theory. J. Amer. Math. Soc. 18 (2005), no. 3, 501-545. MR2138135(2006e:19004)

[BR07] Bartels, Arthur; Reich, Holger: Coefficients for the Farrell-Jones conjecture. Adv. Math. 209 (2007), no. 1, 337-362. MR2294225 (2008a:19002)

[DL98] Davis, James F.; Lück, Wolfgang: Spaces over a category and assembly maps in isomorphism conjectures in $K$ - and $L$-theory. $K$-Theory 15 (1998), no. 3, 201-252. MR1659969 (99m:55004)

[LR05] Lück, Wolfgang; Reich, Holger: The Baum-Connes and the Farrell-Jones conjectures in $K$ - and $L$-theory. Handbook of $K$-theory. Vols. 1, 2, 703-842, Springer, Berlin, 2005. MR 2181833 (2006k:19012)

Mathematisches Institut, Universität Bonn, Endenicher Allee 60, Bonn, D-53115, GERMANY

E-mail address: wegner@math.uni-bonn.de 